\title{
Application of Fabry perot interferometer and error analysis
}

\author{
Tian Yang \\ BeiHang University XueYuan Road No.37,HaiDian District,BeiJing,China \\ wqggmfswl@buaa.edu.cn
}

Keywords: sodium wavelength difference, F-p interference, multi-beam interference

Abstract. According to the theory of multiple-beam interference, the difference of sodium wavelength can be measured by using fabry-perot interferometer. After experiment, unary linear regression and uncertainly calculation has been down based on the raw data. At last we do qualitative and quantitative error analysis by our own sights.

\section{Introduction.}

Fabry perot interferometer (Fig.1) makes use of multiple reflections between two closely spaced partially silvered surfaces. Part of the light is transmitted each time the light reaches the second surface, resulting in multiple offset beams which can interfere with each other. The large number of interfering rays produces an interferometer with extremely high resolution, somewhat like the multiple slits of a diffraction grating increase its resolution.All manuscripts must be in English, also the table and figure texts, otherwise we cannot publish your paper.

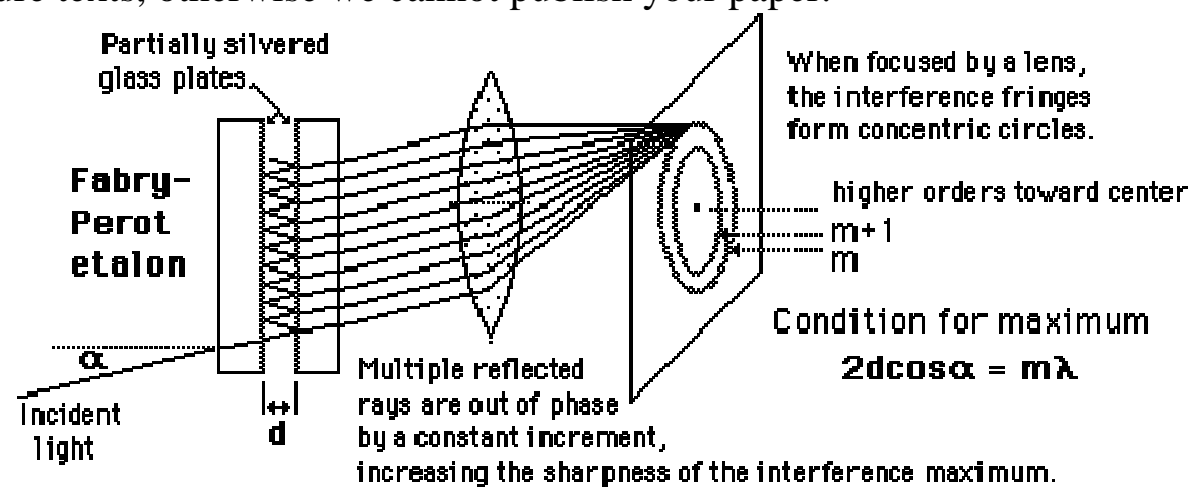

Fig. 1 Fabry perot interferometer

\section{Measuring the differences of sodium wavelength.}

We use sodium lamp as lighting source, then make sure reflectors P1 and P2 on F-P interferometer are strictly parallel. After that, fringes of equal inclination would be found on the screen. After a series of calculation, we finally get the result of wavelength differential between sodium lamp.

The experiment bases on the theory of multi-beam interference. Stripe nesting is our standard to confirm the wavelength differential between sodium lamp. We assume that wavelength of sodium lamp is $\lambda_{1}$ and $\lambda_{2}$, while $\lambda_{1}>\lambda_{2}$. When the fringes get nested, we assume that interference fringe of $\lambda_{1}$ named level $\mathrm{k}_{1}$ and interference fringe of $\lambda_{2}$ named level $\mathrm{k}_{2}+1$. We have the result that

$2 \mathrm{~d} \cos \theta=\mathrm{k}_{1} \lambda_{1}=\left(\mathrm{k}_{2}+0.5\right) \lambda_{2}$

d means thickness of the air layer.

While $\mathrm{d} \rightarrow \mathrm{d}+\Delta \mathrm{d}$, we find the nester phenomenon again. If $\mathrm{k}_{1} \rightarrow \mathrm{k}_{1}+\Delta \mathrm{k}$ now, so $\mathrm{k}_{2}+0.5 \rightarrow \mathrm{k}_{2}+0.5+\Delta \mathrm{k}+1$.

We have the result that

$$
2(\mathrm{~d}+\Delta \mathrm{d}) \cos \theta=\left(\mathrm{k}_{1}+\Delta \mathrm{k}\right) \lambda_{1}=\left(\mathrm{k}_{2}+0.5+\Delta \mathrm{k}+1\right) \lambda_{2}
$$

Subtract from equation (1) and equation 2 then we get the result 
$2 \Delta \mathrm{d} \cos \theta=\Delta \mathrm{k} \lambda_{1}=(\Delta \mathrm{k}+1) \lambda_{2}$

So

$1 / \Delta \mathrm{k}=\lambda_{1} /(2 \Delta \mathrm{d} \cos \theta), \quad \lambda_{1}-\lambda_{2}=\lambda_{2} / \Delta \mathrm{k}$

So

$$
\Delta \lambda=\lambda_{1}-\lambda_{2}=\left(\lambda_{1} \lambda_{2}\right) /(2 \Delta \mathrm{d} \cos \theta) \approx \bar{\lambda}^{2} /(2 \Delta \mathrm{d})
$$

We use F-P interferometer to accomplish the experiment. It is modified by Michael interferometer. While P2 plate position is fixed, P1 can be moved by turning the rough wheel or the handwheel to change the spacing of the plate. Recording $\mathrm{p} 1$ 's position as $\mathrm{d} 1$ when nested phenomenon appeared, then moving $\mathrm{p} 1$ and repeat the operation 10 times.

\section{Data processing.}

Table1 Position of $\mathrm{P}_{1}$

\begin{tabular}{|c|c|c|c|c|c|}
\hline $\mathrm{i}$ & 1 & 2 & 3 & 4 & 5 \\
\hline$d_{i} / \mathrm{mm}$ & 28.94101 & 29.22619 & 29.50848 & 29.79770 & 30.08108 \\
\hline $\mathrm{i}$ & 6 & 7 & 8 & 9 & 10 \\
\hline$d_{i} / \mathrm{mm}$ & 30.37569 & 30.70042 & 30.98622 & 31.29002 & 31.58570 \\
\hline
\end{tabular}

From the introduction

$$
\mathrm{d}_{\mathrm{i}}=\frac{\bar{\lambda}^{2}}{2 \Delta \lambda} \mathrm{i}+\mathrm{d}_{0}
$$

let

$$
\mathrm{i} \equiv \mathrm{X}, \mathrm{d}_{\mathrm{i}} \equiv \mathrm{Y}, \mathrm{b} \equiv \frac{\bar{\lambda}^{2}}{2 \Delta \lambda}
$$

based on the raw data

$$
\bar{X}=5.5, \bar{Y}=3.0248 * 10^{-2} m
$$

so

$$
\overline{X^{2}}=38.5, \overline{Y^{2}}=9.156779 * 10^{-4} \mathrm{~m}^{2}, \overline{X Y}=0.1687936 \mathrm{~m}
$$

so

$$
\mathrm{b}=\frac{\overline{X Y}-\bar{X} \bar{Y}}{\overline{X^{2}}-\bar{X}^{2}}=0.2943 * 10^{-3} \mathrm{~m}
$$

The standard sodium wavelength that we use is $589.3 * 10^{-9} \mathrm{~m}$

So

$$
\Delta \lambda=5.900008325 * 10^{-10} \mathrm{~m}
$$

The correlation coefficient is

$$
\mathrm{r}=\frac{\overline{X Y}-\bar{X} \bar{Y}}{\sqrt{\left(\bar{X}^{2}-\overline{X^{2}}\right)\left(\bar{Y}^{2}-\overline{Y^{2}}\right)}}=0.9998 \approx 1
$$

Therefore, the curve is highly linearly dependent. And then we calculated the uncertainty of $\Delta \lambda$ A type uncertainty of linear regression coefficient $b$ is 


$$
u_{a}(b)=b \sqrt{\frac{1}{k-2}\left(\frac{1}{r^{2}}-1\right)}=1.47164 * 10^{-6} m
$$

B type uncertainty of linear regression coefficient $b$ is

$$
u_{b}(b)=\frac{0.00005}{\sqrt{3}} \mathrm{~mm}=2.8867 * 10^{-8} \mathrm{~m}
$$

Uncertainly of linear regression coefficient $b$ is

$$
u(b)=\sqrt{u_{a}^{2}(b)+u_{b}^{2}(b)}=1.4717 * 10^{-6} m
$$

Uncertainly of $\Delta \lambda$ is

$$
\mathrm{u}(\Delta \lambda)=-\Delta \lambda \frac{\mathrm{u}(\mathrm{b})}{\mathrm{b}}=-2.9927112 * 10^{-12} \mathrm{~m}
$$

So, the result is expressed as

$$
\Delta \lambda \mp u(\Delta \lambda)=(5.90 \mp 0.03) \times 10^{-10} \mathrm{~m}
$$

Error analysis. We mainly discuss the influence of $\Delta \lambda$ from selection of di's position .It is obviously that $\mathrm{P}_{1}$ and $\mathrm{P}_{2}$ is not strictly parallel. We assume that the angle between $\mathrm{P}_{1}$ and $\mathrm{P}_{2}$ is $\alpha$. When length become $\mathrm{P}_{1}$ and $\mathrm{P}_{2}$, named $\mathrm{d}$, become larger, the force binding between $\mathrm{P}_{2}$ and guideway become worse, that means $\alpha$ would become larger because the device is in poor parallelism now.

According to $\Delta \mathrm{d}=\frac{\lambda^{2}}{2 \Delta \lambda \cos \alpha}, \Delta \mathrm{d}$ will become bigger when $\alpha$ gets larger. To prove the derivation above, we take two empirical data that we have done before.

Table 2

\begin{tabular}{|l|r|r|r|r|r|r|r|r|r|r|}
\hline $\mathrm{i}$ & 1 & 2 & 3 & 4 & 5 & 6 & 7 & 8 & 9 & 10 \\
\hline $\mathrm{di}$ & 28.94101 & 29.22619 & 29.50848 & 29.7977 & 30.08108 & 30.37569 & 30.68042 & 30.98622 & 31.29002 & 31.5857 \\
\hline $\mathrm{di}+1 \cdot \mathrm{di}$ & 0.28518 & 0.28229 & 0.28922 & 0.28338 & 0.29461 & 0.30473 & 0.3058 & 0.3038 & 0.29568 & \\
\hline $\mathrm{di}^{\prime} \cdot \mathrm{2}$ & 26.87529 & 27.16703 & 27.45817 & 27.74742 & 28.03438 & 28.32975 & 28.62105 & 28.91017 & 29.20575 & 29.49214 \\
\hline $\mathrm{di}^{\prime} \mathrm{I}^{\prime} \cdot \mathrm{di} \mathrm{i}^{\prime}$ & 0.28374 & 0.29114 & 0.28925 & 0.28996 & 0.29537 & 0.2913 & 0.28912 & 0.29558 & 0.29639 & \\
\hline
\end{tabular}

Then we make the figure by $d_{i}$ and $d_{i+1}$

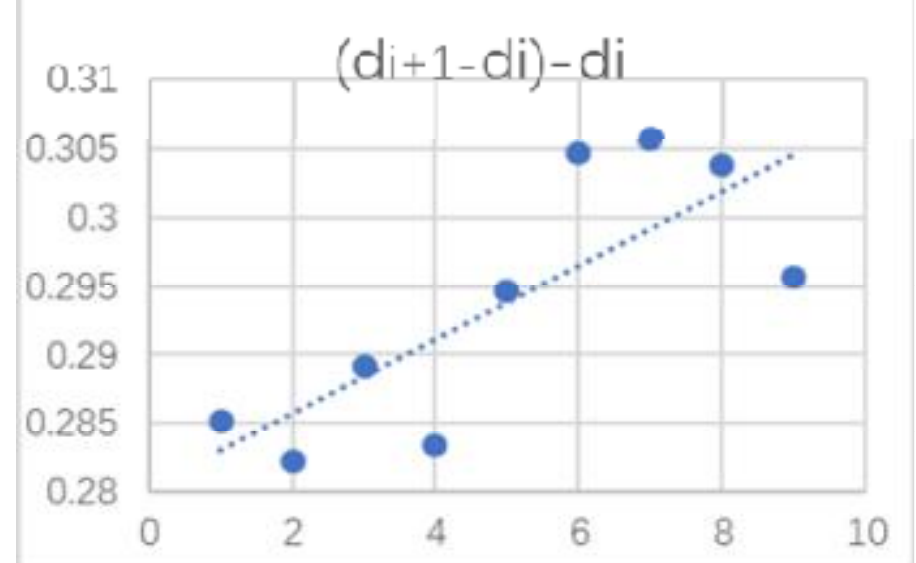

Fig 2. Relationship between $d_{i}$ and $d_{i+1}-d_{i}$ 


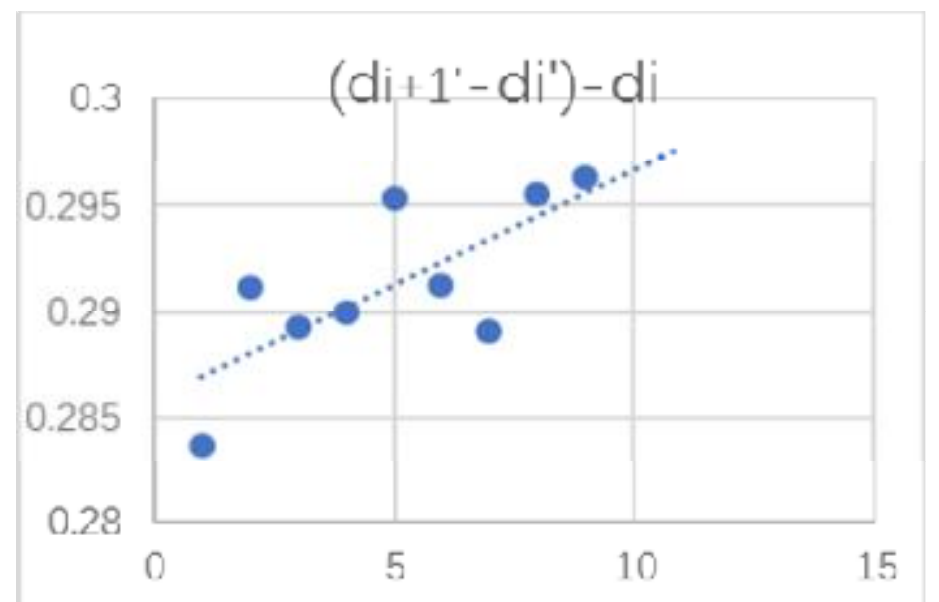

Fig 3. Relationship between $d_{i}$ ' and $d_{i+1}$ ' $-d_{i}$ '

\section{Conclusions.}

At first, from the figure, we can see that when $\mathrm{d}_{\mathrm{i}}$ becomes large, $\Delta \mathrm{d}$ also become large. Secondly, comparing di from two tables, we find that $\Delta \mathrm{d}>\Delta \mathrm{d}$ ' while di $>$ di'. So the theoretical predictions confirmed.

\section{References}

[1] Chaorong Li: Basic physics experiment (revised) (in Chinese), BeiHang University Press.

[2] Li Wang, Yi Zhou ,Dengxin Hua: Research and simulation of atmospheric wind field and temperature field detection based on fabry-perot interferometer(in Chinese), $[\mathrm{J}]$. Acta Optica Sinica, 2011 (10): 1-6.

[3] Baohong Ma, Zhanjie Zhang: Principle and application analysis of fabry perot interferometer(in Chinese), [J] . Journal of Luoyang Normal University, 2012 (11) : 29-30.

[4] Jiansu Liu, Yingen He: The technique and application of farbre-perot (f-p) interferometer(in Chinese), Academic exchange 